\title{
Higher potency statins and the risk of new diabetes: multicentre, observational study of administrative databases
}

\author{
(c) $(1)$ (8) OPEN ACCESS
}

\author{
Colin R Dormuth assistant professor ${ }^{1}$, Kristian B Filion assistant professor ${ }^{2}$, J Michael Paterson \\ scientist ${ }^{3}$, Matthew $\mathrm{T}$ James assistant professor ${ }^{4}$, Gary $\mathrm{F}$ Teare director of measurement and \\ analysis ${ }^{5}$, Colette B Raymond research scientist ${ }^{6}$, Elham Rahme associate professor ${ }^{7}$, Hala Tamim \\ associate professor ${ }^{8}$, Lorraine Lipscombe adjunct scientist ${ }^{3}$, for the Canadian Network for \\ Observational Drug Effect Studies (CNODES) Investigators
}

\begin{abstract}
${ }^{1}$ Department of Anesthesiology, Pharmacology and Therapeutics, University of British Columbia, Victoria, BC V8W 1Y2, Canada; ${ }^{2}$ Centre for Clinical Epidemiology, Lady Davis Institute, Jewish General Hospital, McGill University, Montreal, Canada; ${ }^{3}$ Institute for Clinical Evaluative Sciences, Toronto, Canada; ${ }^{4}$ Department of Medicine, University of Calgary, Calgary, Canada; ${ }^{5} \mathrm{Health}$ Quality Council, Saskatoon, Canada; ${ }^{6}$ Manitoba Centre for Health Policy, University of Manitoba, Winnipeg, Canada; ${ }^{7}$ Department of Medicine, McGill University, Montreal, Canada; ${ }^{8}$ School of Kinesiology and Health Science, York University, Toronto, Canada
\end{abstract}

\begin{abstract}
Objective To evaluate the incremental increase in new onset diabetes from higher potency statins compared with lower potency statins when used for secondary prevention.

Design Eight population based cohort studies and a meta-analysis.

Setting Six Canadian provinces and two international databases from the UK and US.

Participants 136966 patients aged $\geq 40$ years newly treated with statins between 1 January 1997 and 31 March 2011.

Methods Within each cohort of patients newly prescribed a statin after hospitalisation for a major cardiovascular event or procedure, we performed as-treated, nested case-control analyses to compare diabetes incidence in users of higher potency statins with incidence in users of lower potency statins. Rate ratios of new diabetes events were estimated using conditional logistic regression on different lengths of exposure to higher potency versus lower potency statins; adjustment for confounding was achieved using high dimensional propensity scores. Meta-analytic methods were used to estimate overall effects across sites.
\end{abstract}

Main outcome measures Hospitalisation for new onset diabetes, or a prescription for insulin or an oral antidiabetic drug.

Results In the first two years of regular statin use, we observed a significant increase in the risk of new onset diabetes with higher potency statins compared with lower potency agents (rate ratio 1.15, 95\% confidence interval 1.05 to 1.26). The risk increase seemed to be highest in the first four months of use (rate ratio $1.26,1.07$ to 1.47).

Conclusions Higher potency statin use is associated with a moderate increase in the risk of new onset diabetes compared with lower potency statins in patients treated for secondary prevention of cardiovascular disease. Clinicians should consider this risk when prescribing higher potency statins in secondary prevention patients.

\section{Introduction}

Labels on statin medications in the United States now include information concerning glycaemic effects, including diabetes and increases in haemoglobin $\mathrm{A}_{1 \mathrm{c}}$ or fasting plasma glucose. The US Food and Drug Administration approved these labelling changes in February 2012, based mainly on evidence drawn from two meta-analyses of randomised controlled trials. ${ }^{1}$ The first meta-analysis of statins compared with placebo, conducted by Rajpathak and colleagues, ${ }^{2}$ included 57593 patients from six trials and showed a small increase in risk for type 2 diabetes (relative risk 1.13, 95\% confidence interval 1.03 to 1.23 ). The second meta-analysis, reported the following year by Sattar and colleagues, examined the effect of statins on the risk of diabetes in 91140 patients from 13 trials. $^{3}$ That meta-analysis showed that statins were associated with a $9 \%$ increased risk of diabetes (odds ratio 1.09, 1.02 to 1.17). Preiss and colleagues also compared the risk of diabetes associated with higher potency 
and lower potency statins in a meta-analysis of 32752 patients in five trials, ${ }^{4}$ and found that higher potency statins were associated with a $12 \%$ increased risk of diabetes relative to lower potency statins (odds ratio 1.12, 1.04 to 1.22).

The meta-analysis by Preiss et al is of particular relevance to patients treated with statins for secondary prevention of cardiovascular events. Strong, clinically meaningful evidence that shows statins reduce all cause mortality in secondary prevention means that balancing treatment risks with benefits reduces to a question of the potency (and perhaps other properties) of which statin to prescribe rather than a choice between treatment or no treatment. Meta-analyses of statins and total mortality in primary prevention patients have provided conflicting results. ${ }^{5-7}$ While statins are recommended for secondary prevention of cardiovascular events in all patients who tolerate treatment, the incremental increase in diabetes risk with higher potency statins compared with lower potency statins remains to be determined.

Existing clinical trials of statins were not based on real world use, were not specifically designed to assess diabetes endpoints (and all had different and informal ways of identifying diabetes), and were not restricted to secondary prevention of cardiovascular events, where the potential benefit-risk profile is different from that for primary prevention. Furthermore, many studies have not been able to ensure that diabetes cases were truly incident, as diabetes is often asymptomatic and more common among those with cardiovascular disease and other indications for statin therapy. Therefore, the Canadian Network for Observational Drug Effect Studies (CNODES) ${ }^{8}$ designed a study to evaluate the risk of new diabetes in patients who received higher potency statins as opposed to lower potency statins shortly after a cardiovascular event or procedure. A priori, we expected to observe an association of similar size for secondary prevention (rate ratio of approximately 1.15), based on the existing meta-analyses conducted in patients receiving statins for primary and secondary prevention. ${ }^{4}$

\section{Methods}

\section{Study population}

We used a common analytical protocol to conduct population based cohort studies of the association between statins and diabetes in six provinces of Canada (Alberta, Manitoba, Nova Scotia, Ontario, Quebec, and Saskatchewan; source population 13.2 million in 2013), as well as in two international databases (the UK Clinical Practice Research Datalink (CPRD), population 11.6 million; and the US MarketScan database, source population about 70 million). The study populations were patients aged $\geq 40$ years, without previously diagnosed or treated diabetes, and newly prescribed a statin (no prescription for a cholesterol lowering drug in the year before hospitalisation) between 1 January 1997 and 31 March 2011 after a hospitalisation for a major cardiovascular event (myocardial infarction, stroke, coronary artery bypass graft, or percutaneous coronary intervention). In three of the Canadian provinces (Alberta, Ontario, and Nova Scotia) data were available only for patients aged $\geq 65$ years, and in the MarketScan database only data for patients aged $<65$ years were available. In four sites patient accrual began after 1997.

\section{Data sources}

All eight participating sites analysed data from their respective administrative healthcare databases, all of which have been used previously for observational research. ${ }^{9}{ }^{10}$ All databases included information on the specific drug dispensed in a prescription, the quantity or days' supply of medication dispensed (or prescribed in CPRD), and the date of dispensing. In general, physician claims and hospitalisation discharge records included date of service encounter or hospital admission, up to 25 ICD-9 or ICD-10 (international classification of diseases, ninth or 10th revision) diagnosis codes, and procedure and billing codes. Patients who were missing demographic data (sex, $<1 \%$; age, $<1 \%$ ) were not included in the analysis, and no imputation methods were used. We assumed that the completeness and misclassification of diagnostic coding in our datasets were similar to those in other administrative databases.

\section{Cohorts of statin treated patients}

We conducted as-treated analyses using nested case-control methodology. This type of analytical approach is well suited to studies involving time dependent exposures, and it provides good computational efficiency for analysing rare events in large databases. ${ }^{11}$ Analyses were undertaken separately at each site according to a common analytical protocol and then meta-analytic methods were used to estimate an overall rate ratio. We assembled secondary prevention cohorts, which included patients who were admitted to a hospital between 1 January 1998 (or one year after the beginning of data availability) and 31 March 2011, who received a coded diagnosis (principal or secondary) for acute myocardial infarction or stroke or a procedure for coronary artery bypass graft or percutaneous coronary intervention during their stay in hospital, who had no record of a diabetes diagnosis during their hospitalisation, and who then received a statin prescription within 90 days after being discharged, having not been prescribed a cholesterol lowering drug in the previous year (World Health Organization Anatomical Therapeutic Class C10, cerivastatin excluded). Patients were required to have a length of stay in hospital for their cohort-defining event or procedure of at least three days and no greater than 30 days. Diagnostic codes for myocardial infarction and stroke and procedural codes for coronary artery bypass graft and percutaneous coronary intervention are listed in the data supplement appendix.

Patients' cohort entry dates were defined as the date of the first statin prescription after discharge from hospital. We excluded patients if they were $<40$ years old (or $<66$ years old in jurisdictions that only had drug data on senior citizens), had less than one year of database history, received any cholesterol lowering drug, or received any diabetes medication or a diabetes diagnosis in the previous year, including diagnoses during their index hospitalisation (drug use in hospital was not available). Our categorisation of daily statin dose was derived from a systematic review and meta-analysis of clinical trials which quantified the effects of statins on serum low density lipoprotein (LDL) cholesterol concentration. ${ }^{12}$ We defined rosuvastatin $\geq 10 \mathrm{mg}$, atorvastatin $\geq 20 \mathrm{mg}$, and simvastatin $\geq 40 \mathrm{mg}$ as higher potency statins, and all other statins were defined as lower potency statins. That analysis showed that statins clustered around three levels of reduction of LDL cholesterol concentration: one group produced an approximate $35 \%$ reduction, one reduced concentration by about $45 \%$, and rosuvastatin $80 \mathrm{mg}$ lowered LDL concentration by $60 \%$. This classification is similar to ones used by CNODES and others. ${ }^{13-15}$ We grouped rosuvastatin $80 \mathrm{mg}$ with the middle group since there were relatively few such prescriptions. Therefore, statins were categorised as higher or lower in our study according to whether they would produce a theoretical $<45 \%$ or $\geq 45 \%$ reduction in LDL cholesterol concentration. 


\section{Diabetes endpoint}

We defined our diabetes endpoint as the first occurrence of a hospitalisation with a principal or secondary diagnosis for diabetes (ICD-9 diagnostic code 250; ICD-10 codes E10, E11, $\mathrm{E} 12, \mathrm{E} 13, \mathrm{E} 14)$ or a prescription for insulin or an oral antidiabetic medication. These were most likely to be incident cases, as most patients admitted for a cardiovascular event would have been screened for diabetes, and anyone with a diabetes diagnosis at that hospital admission was excluded. Using hospital discharge data to capture diabetes was previously evaluated to have $90 \%$ sensitivity and $92 \%$ specificity in the Canadian province of Ontario. ${ }^{16}$

\section{High dimensional propensity scores}

We estimated high dimensional propensity scores for all patients at each site. The high dimensional propensity score algorithm is available as downloadable SAS software files from the Brigham and Women's Hospital. ${ }^{17}$ As described in detail elsewhere, ${ }^{18}$ the algorithm prioritises thousands of drug, diagnostic, procedure, and demographic variables according to their potential to cause bias in the estimate of an exposure-outcome association (such as rate ratio). Typically, the 200-500 variables most likely to cause bias are included in a propensity score model that is estimated using logistic regression. In our analysis, we used high dimensional propensity scores to estimate the predicted probability (propensity score) of exposure to higher potency statins versus lower potency statins, conditional on all of the included covariates.

In addition to the 500 covariates empirically selected for the propensity score models, we also included the following pre-specified covariates: year of cohort entry and, from the year preceding cohort entry, binary indicator variables for whether the patient was hospitalised, had a laboratory test, received more than four distinct prescription drugs dispensed, received a loop diuretic, had more than four physician visits, or had a diagnosis for hypertensive disease, hypercholesterolemia, peripheral vascular disease, or congestive heart failure. After estimating propensity scores, we trimmed from the analysis the patients with the smallest $5 \%$ and largest $5 \%$ of propensity scores.

\section{As-treated analysis}

A follow-up end date was defined for each patient as the earliest occurrence of a diabetes endpoint, date of death, date of emigration, date of loss of continuous health or drug plan enrolment, 24 months after start of statin treatment, a dispensing for cerivastatin, or 31 March 2011. All cases of the diabetes endpoint that occurred during follow-up were identified, and the date of the event was defined as the index date. Ten controls were randomly selected for each case from the risk set of patients who remained at risk of diabetes, after matching on sex, age (within two years), and a requirement to have entered the cohort within 90 days of the cohort entry date of the case.

We used conditional logistic regression to estimate matched odds ratios between patients treated with higher potency statins and those treated with lower potency statins for up to two years of statin exposure. Adjusted matched odds ratios were estimated by including tenth of propensity score (nine binary indicator variables) and type of event for which the patient entered the cohort (three binary indicator variables for stroke, coronary artery bypass graft, and percutaneous coronary intervention; myocardial infarction was the reference category). Because of our method for sampling controls, odds ratios from our conditional logistic regressions were equivalent to rate ratios. In addition, we examined three mutually exclusive durations of current exposure within the two year exposure timeframe $(\leq 120$ days, 121-365 days, and 366-730 days). Rate ratios of diabetes were compared between higher and lower potency statin-treated patients within each duration category. Patients who received both a higher and lower potency statin in the same current exposure category were categorised as higher potency patients in that particular category.

Given that the mechanism by which statins might cause diabetes is unknown, we conducted a sensitivity analysis in which patients meeting our diabetes definition within the first 90 days of statin treatment were censored ( 90 days and 180 days at the Ontario site); in effect assuming that early events could not be the result of treatment. Thus, events included in the sensitivity analysis in the current cumulative exposure categories were events that occurred at least 90 days after exposure to statins. Patients who had events in the first 90 days were censored from further follow-up and could not serve as controls at a later time, as was the case with any patient who had an event. This sensitivity analysis was the same as the main analysis in all other respects.

\section{Meta-analysis of site-specific results}

The data analysts at each site were kept blind to the results of other sites until after the meta-analysis was completed. For the meta-analysis, we pooled the results from each site using fixed effects and random effects models. Inverse variance weighted rate ratios and $95 \%$ confidence intervals were calculated to estimate the overall effect across sites for each pre-specified duration of statin exposure.

\section{Results}

\section{Study populations}

Overall, there were in excess of two million patients in participating databases newly exposed to statins during the study period. After restricting the analysis to patients newly dispensed statins within 90 days of a hospitalisation for myocardial infarction, stroke, coronary artery bypass graft, or percutaneous coronary intervention, and after trimming the $10 \%$ of patients with the most extreme propensity scores, there were 136936 patients who remained eligible for further analysis. Baseline characteristics of the overall study population, matched and unmatched on propensity score, are shown in table $1 \Downarrow$, demonstrating the comparability of patients prescribed higher potency statins and those prescribed lower potency statins in measured factors conditional on propensity score. The mean age of study participants was 68 years, and $63 \%$ were men.

\section{Diabetes events}

Rates of our diabetes endpoint in Canadian provinces within two years of starting a statin were between 2.12/100 patients in the Nova Scotia study population and 3.40/100 patients in Saskatchewan. The event rate in the US MarketScan study population was 2.99/100, and in the UK CPRD it was 1.95/100. In total, there were 3629 cases of new onset diabetes in the first two years of follow-up in our study population of 136966 patients. Table $2 \Downarrow$ describes the characteristics of these cases of new diabetes and their matched controls. Cases had slightly greater prevalence of congestive heart failure and hypertensive disease (and drug use for those conditions) compared with their controls. 


\section{As-treated analyses}

The figure $\Downarrow$ shows rate ratios from a fixed effect analysis for our diabetes endpoint in patients who started higher potency statins compared with patients who started lower potency statins. For current cumulative exposure within two years, we observed a $15 \%$ higher rate of diabetes in patients prescribed higher potency statins compared with patients prescribed lower potency statins (fixed effect rate ratio $1.15,95 \%$ confidence interval 1.05 to 1.26 ). We estimated that 342 secondary prevention patients needed to be treated with a higher potency statin instead of a lower potency statin for two years to cause one additional case of diabetes. Excluding Alberta and Nova Scotia, two provinces that showed large protective effects, the overall rate ratio for up to two years of therapy was 1.20 (95\% confidence interval 1.09 to 1.31 ). The risk increase seemed to be highest in the first four months of statin use. For $\leq 120$ days of exposure, we observed a $26 \%$ relative increase in patients prescribed higher potency statins compared with patients prescribed lower potency statins (fixed effect rate ratio $1.26,95 \%$ confidence interval 1.07 to 1.47 ). A similar rate ratio was observed in the 121-365 day exposure category (fixed rate ratio $1.19,1.02$ to 1.38 ). Rate ratios were closer to the null in the 366-730 day exposure category (rate ratio $1.08,0.93$ to 1.25$)$. When Alberta and Nova Scotia were excluded, the overall rate ratio was 1.36 (1.15 to 1.60 ) for the $\leq 120$ day category, 1.22 (1.04 to 1.42) for the 121-365 day exposure category, and $1.11(0.95$ to 1.29$)$ for the 366-730 day category.

The overall association was attenuated and less precise under random effects analysis compared with the fixed effect analysis. For current cumulative exposure within two years, we observed a non-significant $11 \%$ increase in diabetes in patients prescribed higher potency statins compared with patients prescribed lower potency statins (random effects rate ratio $1.11,95 \%$ confidence interval 0.96 to 1.27). Excluding Alberta and Nova Scotia, the overall rate ratio for up to two years of therapy was 1.20 (1.09 to 1.31 ). For $\leq 120$ days of exposure, we observed a non-significant $17 \%$ relative increase (random effects rate ratio $1.17,0.90$ to 1.53 ). A similar rate ratio was observed in the 121-365 day exposure category.

We conducted a sensitivity analysis in which patients with a recorded diabetes event in the first 90 days of statin treatment were not counted as cases and instead were censored from further analysis. Accordingly, the number of cases in the $\leq 120$ day exposure category was reduced to 269 cases, compared with 945 in the main analysis. The association in that exposure category was attenuated compared with the main analysis (rate ratio $1.11,0.85$ to 1.47 ) but remained significantly elevated in the 121-365 day exposure category (rate ratio $1.23,1.05$ to 1.44 ). At the Ontario site, the largest site in the network, a further variation was conducted where diabetes events in the first 180 days of statin treatment were excluded as cases. Again, the event rate was relatively greater in patients prescribed higher potency statins (rate ratio for 121-365 day exposure in Ontario 1.46, 1.03 to 2.07$)$.

\section{Discussion}

In this study of over 136936 patients hospitalised for a major cardiovascular event or procedure, a group in which statin treatment is strongly indicated for secondary prevention, we observed a moderately increased risk of new onset diabetes in patients prescribed higher potency statins compared with lower potency statins. Although the increased risk of diabetes is small and somewhat imprecise, the risk warrants careful consideration given that randomised controlled trials making head to head comparisons of higher potency and lower potency statins in patients with stable coronary heart disease showed no difference in all cause mortality (odds ratios between 0.98 and 1.01$)^{19} 20$ or serious adverse events (which includes cardiovascular events; relative risk 1.00). ${ }^{21}$ Multiple trials have shown that the risk of all cause mortality is reduced in patients treated with a statin for secondary prevention of cardiovascular events instead of placebo, but the risk of all cause mortality was equally likely in trials that directly compared higher potency and lower potency statins in patients with stable coronary heart disease. ${ }^{19-22}$ Serious adverse events were also equally likely, and withdrawals from treatment due to adverse events were more likely in patients prescribed higher potency statins. ${ }^{22}$

We conducted both fixed and random effects analyses. On an empirical basis, the results of some $\chi^{2}$ tests for heterogeneity would suggest that some of the exposure categories could be analysed assuming random effects. Random effects analysis assumes that the studies were drawn from populations that differ from each other in ways that could affect the treatment effect. Fixed effect analysis assumes that all studies in the meta-analysis share a common true effect size. Our site-specific estimates were adjusted for age, sex, and several other potential confounders using high dimensional propensity scores. We believe that the fixed effect analysis is more valid given our statistical adjustments and the fact that we used a common analytical protocol at all sites.

\section{Comparison with existing evidence}

The overall rate ratio for current treatment that we observed in our analysis of statins and diabetes (rate ratio 1.15) was similar to the relative risks reported in the meta-analyses by Rajpathak et al (relative risk 1.13), ${ }^{2}$ by Sattar et al (odds ratio 1.09), ${ }^{3}$ and by Preiss et al in a meta-analysis of trials comparing higher potency and lower potency statins (odds ratio 1.12). ${ }^{4}$ Our rate ratios are also close to hazard ratios reported in another Canadian observational study. ${ }^{23}$ Our results differ from other observational studies that have reported either no significant association ${ }^{24}$ or multi-fold increases in relative risk. ${ }^{25} 26$ The proximity of our results with our a priori hypothesis, and their agreement with the maximum likelihood estimates observed in most other studies, together lend meaningful support for an increased risk of diabetes associated with higher potency statins compared with lower potency statins.

Some researchers have argued that a potential absolute increase in new onset diabetes with high dose statins compared with low dose statins is outweighed by a larger reduction in cardiovascular events. ${ }^{27}{ }^{28}$ A meta-analysis of randomised controlled trials lends support to this argument, ${ }^{4}$ the basis for which comes from trials specifically designed to measure cardiovascular events but where diabetes events were not recorded in a consistent or required way. In fact, most diabetes events in the trials in question relied on physicians' adverse event reports, which are often underreported. Our study was partially motivated by these aspects of the trials of higher potency versus lower potency statins.

Our study was specifically designed to examine a hypothesis that the risk of new onset diabetes was associated with statin potency. While there are several plausible mechanisms consistent with a potency hypothesis, ${ }^{29}$ other distinct hypotheses have also been advanced, such as an effect related to the hydrophylic/lipophylic status of each statin, or a statin-specific effect. Our results do not lend support to a hydrophylic/lipophylic hypothesis because the two hydrophylic statins (pravastatin and rosuvastatin) are at near opposite ends 
of the potency spectrum in their effect on serum LDL cholesterol. Our results are somewhat compatible with other research that showed an increased diabetes risk with rosuvastatin, atorvastatin, and simvastatin. ${ }^{23}$ Although potency and statin-specific mechanisms are distinctly different hypothesis, the results might be expected to be compatible because the specific statins associated with an increased risk above are also the three most potent statins.

\section{Methodological considerations}

Our diabetes outcome definition, consisting of a diagnosis of diabetes in a hospital admission or a prescription for a diabetes medication, probably did not capture some patients diagnosed in an ambulatory setting but not treated with pharmacotherapy. Ascertaining diabetes from physician claims data typically requires multiple visits over a number of months in order to remove false positives. We excluded such potential cases from our outcome definition to avoid potential bias in the rate ratio due either to exposure misclassification (had we defined such ambulatory cases on the second or third visit for diabetes) or immortal time bias (after multiple visits going back to the first visit to define the index date). Some of the excluded cases could have been selected as controls, but this should have been infrequent and therefore of little consequence given the large pool of controls.

Although our outcome definition omitted some cases, we did not expect that our definition would be different between patients prescribed higher potency or lower potency statins. Our definition probably captured patients with more serious disease because they were either admitted to hospital or required medication. Furthermore, patients included in our study were all hospitalised for an occlusive vascular event or procedure, and those patients would have likely undergone serum blood glucose testing while in hospital. While provinces and hospitals vary in how well secondary diagnoses are captured, the fact that all patients in the study were hospitalised before starting their statin should have substantially eliminated prevalent diabetes cases at cohort entry.

Rigorous definitions for identifying diabetes patients in administraive claims for physician office visits typically require multiple such visits over time, in order to rule out cases where diabetes was suspected but where the disease was not confirmed in subsequent testing. Using multiple office visits to identify ambulatory cases in an as-treated analysis would have required the introduction of immortal person time into the design ${ }^{30}$ hence those potential cases did not meet the study outcome definition. However, diagnoses for diabetes recorded in physician office visits were still used to exclude patients from entering the secondary prevention cohorts, to minimise the risk that the patients who entered the study were not free of diabetes at baseline. More importantly, there was no diabetes diagnosis on the hospital discharge abstract for all patients who entered the study.

In administrative claims data, as in clinical practice, it is usually impossible to determine the exact timing of the onset of diabetes. As with many other diseases in observational research, the date of a first encounter with the healthcare system that coincides with the first recorded occurrence of the disease is used as a proxy for the timing of disease onset. Further, our endpoint was defined as the date of a first antidiabetic prescription in many cases, which would have required a prior diagnosis of diabetes. Given the possibility that some cases in our study might have had undiagnosed diabetes when they started taking a statin, we conducted a sensitivity analysis in which diabetes cases captured in the first 90 days of treatment were not counted. This alternative approach effectively eliminated most cases in the $\leq 120$ day category of current cumulative statin exposure. Still, diabetes risk remained significatly elevated in patients prescribed higher potency statins in the 121-365 day exposure category. This sensitivity analysis provided some reassurance that our rate ratios were capturing a true relative association.

Even though our results are much more compatible with the maximum likelihood estimates reported in previous meta-analyses than with a null hypothesis, confounding by indication remains a possible threat to the validity of our results. To minimise this bias, our reference group consisted of patients who also received a statin. We adjusted for a broad spectrum of possible confounders using high dimensional propensity scores, which included both pre-specified and empirically identified confounders. There was probably a trend over time towards use of higher potency statins, and we therefore included calendar year of cohort entry as a covariate in our propensity score models.

\section{Conclusions}

We found modest evidence that there is a harmful association between statin potency and new diabetes in patients treated for secondary prevention of cardiovascular disease. Clinicians should consider our study results when choosing between lower potency and higher potency statins in secondary prevention patients, perhaps bearing in mind that head to head randomised trials of higher potency versus lower potency statins have not shown a reduction in all cause mortality or serious adverse events in secondary prevention patients with stable disease. ${ }^{19-21}$

This study was made possible through data sharing agreements between CNODES member research centres and the respective provincial governments of Alberta, Manitoba, Nova Scotia, Ontario, Quebec, and Saskatchewan. The opinions, results, and conclusions reported in this paper are those of the authors. No endorsement by the provincial governments is intended or should be inferred. We acknowledge the important contributions of the CNODES collaborators and assistants at each site: Richard Morrow (BC, MarketScan), James Zhang (Alberta), Alomgir Hossain (Saskatchewan), Matthew Dahl (Manitoba), Fangyun Wu (Ontario), Hacene Nedjar (Quebec), Steve Doucette and Yan Wang (Nova Scotia), and Sophie Dell'Aniello (CPRD). We also acknowledge the assistance of Dr James Wright, who provided his expert understanding of randomised trials of statins.

The Canadian Network for Observational Drug Effect Studies (CNODES) Investigators are: Samy Suissa (principal investigator); Colin Dormuth (British Columbia); Brenda Hemmelgarn (Alberta); Gary Teare (Saskatchewan); Patricia Martens and Patricia Caetano (Manitoba); David Henry and Michael Paterson (Ontario); Jacques LeLorier (Québec); Adrian Levy (Nova Scotia); Pierre Ernst (UK General Practice Research Database (CPRD)); Robert Platt (Methods); and Ingrid Sketris (Knowledge Translation).

Contributors: CRD oversaw the development of the study protocol and the creation of a data analysis plan that was adapted for use in each jurisdiction and each dataset. All authors contributed to discussions on protocol development and provided critical revisions to the manuscript. LL defined the diabetes outcome. KBF, JMP, GFT, and HT contributed to study design issues. CRD drafted the manuscript and incorporated co-authors' suggestions, and is the guarantor of the manuscript. The final manuscript was approved by the publications subcommittee of CNODES.

Funding: CNODES is a collaborating centre of the Drug Safety and Effectiveness Network (DSEN), funded by the Canadian Institutes of Health Research (Grant No DSE-111845). The funding source was not 


\section{What is already known on this topic}

Meta-analysis of randomised clinical trials suggest that statins increase the risk of new diabetes.

Statins are recommended for secondary prevention of cardiovascular disease in all patients who tolerate treatment, but the incremental increase in risk of new onset diabetes with higher potency statins as opposed to lower potency statin treatment in this population remains to be determined.

\section{What this study adds}

Our study suggests that use of higher potency statins instead of lower potency statins is associated with a moderate increase in the risk of new onset diabetes in patients treated for secondary prevention of cardiovascular disease.

involved in the writing of the manuscript or the decision to submit it for publication.

Ethical approval: Approval for each study was obtained from the respective academic institutions at each site.

Competing interests: All authors have completed the ICMJE uniform disclosure form at www.icmje.org/coi_disclosure.pdf (available on request from the corresponding author) and declare: no support from any organisation for the submitted work; ER has received grants and consultant fees from Pfizer Canada that were unrelated to this study, and MTJ received an honorarium for a presentation at an industry sponsored conference by Amgen unrelated to this study; no other relationships or activities that could appear to have influenced the submitted work.

Data sharing: CNODES is not permitted to release individual level data or aggregated data with small cell sizes. The scientific protocol for this analysis is available on request.

Transparency: The lead author (the manuscript's guarantor) affirms that the manuscript is an honest, accurate, and transparent account of the study being reported; that no important aspects of the study have been omitted; and that any discrepancies from the study as planned (and, if relevant, registered) have been explained.

1 Food and Drug Administration. FDA drug safety communication: Important safety labe changes to cholesterol-lowering statin drugs. 2012. www.fda.gov/drugs/drugsafety/ ucm293101. htm.

2 Rajpathak SN, Kumbhani DJ, Crandall J, Barzilai N, Alderman M, Ridker PM. Statin therapy and risk of developing type 2 diabetes: a meta-analysis. Diabetes Care 2009;32:1924-9.

3 Sattar N, Preiss D, Murray HM, Welsh P, Buckley BM, de Craen AJ, et al. Statins and risk of diabetes: a collaborative meta-analysis of randomised statin trials. Lancet 2010;375:735-42.

4 Preiss D, Seshasai SR, Welsh P, Murphy SA, Ho JE, Waters DD, et al. Risk of diabetes with intensive-dose compared with moderate-dose statin therapy. JAMA 2011;305:2556-64. Brugts JJ, Yetgin T, Hoeks SE, Gotto AM, Shepherd J, Westendorp RG, et al. The benefits of statins in people without established cardiovascular disease but with cardiovascular risk factors: meta-analysis of randomised controlled trials. BMJ 2009;338:b2376.

6 Ray KK, Seshasai SR, Erqou S, Sever P, Jukema JW, Ford I, et al. Statins and all-cause mortality in high-risk primary prevention: a meta-analysis of 11 randomized controlled trials involving 65,229 participants. Arch Intern Med 2010;170:1024-31.

7 Taylor F, Ward K, Moore THM, Burke M, Davey Smith G, Casas J-P, et al. Statins for the primary prevention of cardiovascular disease. Cochrane Database Syst Rev 2011;(1):CD004816. doi:10.1002/14651858.CD004816.pub3.

8 Suissa S, Henry D, Caetano P, Dormuth CR, Ernst P, Hemmelgarn B, et al. CNODES: the Canadian Network for Observational Drug Effect Studies. Open Med 2012:6:e134-40.

9 Dormuth CR, Hemmelgarn BR, Paterson JM, James MT, Teare GF, Raymond CB, et al. Use of high potency statins and rates of admission for acute kidney injury: multicenter, retrospective observational analysis of administrative databases. BMJ 2013;346:f880.

10 Filion KB, Chateau D, Targownik LE, Gershon A, Durand M, Tamim H, et al. Proton pump inhibitors and the risk of hospitalisation for community-acquired pneumonia: replicated cohort studies with meta-analysis. Gut 2014:63:552-8

11 Essebag V, Platt RW, Abrahamowicz M, Pilote L. Comparison of nested case-control and survival analysis methodologies for analysis of time-dependent exposure. BMC Med Res Methodol 2005;5:5.
12 Law MR, Wald NJ, Rudnicka AR. Quantifying effect of statins on low density lipoprotein cholesterol, ischaemic heart disease, and stroke: systematic review and meta-analysis. BMJ 2003;326:1423.

13 Kheterpal S, Tremper KK, Englesbe MJ, O'Reilly M, Shanks AM, Fetterman DM, et al. Predictors of postoperative acute renal failure after noncardiac surgery in patients with previously normal renal function. Anesthesiology 2007;107:892-902.

14 Ouattara A, Benhaoua H, Le Manach Y, Mabrouk-Zerguini N, Itani O, Osman A, et al. Perioperative statin therapy is associated with a significant and dose-dependent reduction of adverse cardiovascular outcomes after coronary artery bypass graft surgery. $J$ Cardiothorac Vasc Anesth 2009.23.633-8.

15 Molnar AO, Coca SG, Devereaux PJ, Jain AK, Kitchlu A, Luo J, et al. Statin use associates with a lower incidence of acute kidney injury after major elective surgery. J Am Soc Nephrol 2011;22:939-46.

16 Hux JE, Ivis F, Flintoft V, Bica A. Diabetes in Ontario: determination of prevalence and incidence using a validated administrative data algorithm. Diabetes Care 2002;25:512-6.

17 High-dimensional propensity score (hd-PS), Version 1. 2013. www.hdpharmacoepi.org/ download/.

18 Schneeweiss S, Rassen JA, Glynn RJ, Avorn J, Mogun H, Brookhart MA. High-dimensional propensity score adjustment in studies of treatment effects using health care claims data. Epidemiology 2009;20:512-22.

19 Pedersen T, Faergeman O, Kastelein J, Olsson AG, Tikkanen MJ, Holme I, et al. High-dose atorvastatin vs usual-dose simvastatin for secondary prevention after myocardial infarction. JAMA 2005:294:2437-45.

20 LaRosa J, Grundy S, Waters D, Shear C, Barter P, Fruchart JC, et al. Intensive lipid lowering with atorvastatin in patients with stable coronary heart disease. N Engl J Med 2005;352:1425-35.

21 Study of the Effectiveness of Additional Reductions in Cholesterol and Homocystein (SEARCH) Collaborative Group, Armitage J, Bowman L, Wallendszus K, Bulbulia R, Rahimi K, et al. Intensive lowering of LDL cholesterol with $80 \mathrm{mg}$ versus $20 \mathrm{mg}$ simvastatin daily in 12064 survivors of myocardial infarction: a double-blind randomized trial. Lancet 2010;376:1658-69.

22 Therapeutics Initiative. High dose versus standard dose statins in stable coronary heart disease. Therapeutics Letter 2012

23 Carter AA, Gomes T, Camacho X, Juurlink DN, Shah BR, Mamdani MM. Risk of diabetes among patients treated with statins: population based study. BMJ 2013;346:f2610.

24 Ko DT, Wijeysundera HC, Jackevicius CA, Yousef A, Wang J, Tu JV. Diabetes mellitus and cardiovascular events in older patients with myocardial infarction prescribed intensive-dose and moderate-dose statins. Circ Cardiovasc Qual Outcomes 2013;6:315-22.

25 Chen CW, Chen TC, Huang KY, Chou P, Chen PF, Lee CC. Differential impact of statin on new-onset diabetes in different age groups: a population-based case-control study in women from an Asian country. PLoS One 2013:8:e71817.

26 Currie $\mathrm{O}$, Mangin D, Williman J, McKinnon-Gee B, Bridgford P. The comparative risk of new-onset diabetes after prescription of drugs for cardiovascular risk prevention in primary care: a national cohort study. BMJ Open 2013;3:e003475.

27 Bell DS, Dinicolantonio JJ, O'Keefe JH. Is statin-induced diabetes clinically relevant? A comprehensive review of the literature. Diabetes Obes Metab 2013. doi:10.1111/dom. 12254

28 Wang KL, Liu CJ, Chao TF, Chen SJ, Wu CH, Huang CM, et al. Risk of new-onset diabetes mellitus versus reduction in cardiovascular events with statin therapy. Am J Cardiol 2014;113:631-6.

29 Navarese EP, Szczesniak A, Kolodziejczak M, Gorny B, Kubica J, Suryapranata H. Statins and risk of new-onset diabetes mellitus: is there a rationale for individualized statin therapy? Am J Cardiovasc Drugs 2014:14:79-87.

30 Suissa S. Immortal time bias in pharmaco-epidemiology. Am J Epidemiol 2008;167:492-9.

\section{Accepted: 25 April 2014}

\section{Cite this as: BMJ 2014;348:g3244}

This is an Open Access article distributed in accordance with the Creative Commons Attribution Non Commercial (CC BY-NC 3.0) license, which permits others to distribute, remix, adapt, build upon this work non-commercially, and license their derivative works on different terms, provided the original work is properly cited and the use is non-commercial. See: http://creativecommons.org/licenses/by-nc/3.0/. 


\section{Tables}

Table 1| Baseline characteristics of the overall study population (patients newly prescribed higher potency statins and those prescribed lower potency statins during 1997-2011), matched and unmatched on propensity score. Values are numbers (percentages) of patients

\begin{tabular}{|c|c|c|c|c|}
\hline \multirow[b]{2}{*}{ Characteristic } & \multicolumn{2}{|c|}{ Matched cohort ${ }^{\star}$} & \multicolumn{2}{|c|}{ Full cohort } \\
\hline & $\begin{array}{l}\text { Higher potency statins } \\
\qquad(\mathrm{n}=30 \mathrm{843})\end{array}$ & $\begin{array}{l}\text { Lower potency statins } \\
\qquad(n=30843)\end{array}$ & $\begin{array}{l}\text { Higher potency statins } \\
\qquad(\mathrm{n}=89077)\end{array}$ & $\begin{array}{l}\text { Lower potency statins } \\
\qquad(n=47889)\end{array}$ \\
\hline \multicolumn{5}{|l|}{ Age (years): } \\
\hline $40-49$ & 2449 (7.9) & $2451(7.9)$ & $7455(8.4)$ & $3410(7.1)$ \\
\hline $50-59$ & 5834 (18.9) & 5837 (18.9) & $16057(18.0)$ & $7783(16.3)$ \\
\hline $60-64$ & 3852 (12.5) & 3846 (12.5) & $9382(10.5)$ & $5087(10.6)$ \\
\hline $65-69$ & 3415 (11.1) & $3406(11.0)$ & 11527 (12.9) & $6386(13.3)$ \\
\hline $70-74$ & 4350 (14.1) & 4373 (14.2) & $13305(14.9)$ & 7832 (16.4) \\
\hline $75-79$ & $4369(14.2)$ & $4343(14.1)$ & $12745(14.3)$ & $7411(15.5)$ \\
\hline$\geq 80$ & $6574(21.3)$ & 6587 (21.4) & $18606(20.9)$ & $9980(20.8)$ \\
\hline \multicolumn{5}{|l|}{ Sex: } \\
\hline Women & $11926(38.7)$ & $11926(38.7)$ & 32504 (36.5) & $18598(38.8)$ \\
\hline Men & $18917(61.3)$ & $18917(61.3)$ & 56573 (63.5) & $29291(61.2)$ \\
\hline
\end{tabular}

Cohort defining events and

procedures:

\begin{tabular}{|c|c|c|c|c|}
\hline Myocardial infarction & $18628(60.4)$ & $18629(60.4)$ & $60751(68.2)$ & 29155 (60.9) \\
\hline Stroke & $7653(24.8)$ & $7646(24.8)$ & $15668(17.6)$ & $11124(23.2)$ \\
\hline CABG & $3032(9.8)$ & $3042(9.9)$ & $7702(8.6)$ & $4970(10.4)$ \\
\hline PCTA & $3564(11.6)$ & $3564(11.6)$ & $15237(17.1)$ & $5859(12.2)$ \\
\hline \multicolumn{5}{|l|}{ Diagnoses: } \\
\hline Hypertensive disease & $17636(57.2)$ & $17328(56.2)$ & $49010(55.0)$ & 25991 (54.3) \\
\hline Hypercholesterolemia & $9741(31.6)$ & $9700(31.4)$ & $25646(28.8)$ & $15614(32.6)$ \\
\hline Peripheral vascular disease & $1111(3.6)$ & $1125(3.6)$ & $2633(3.0)$ & $1794(3.7)$ \\
\hline Congestive heart failure & $5472(17.7)$ & $5477(17.8)$ & $14523(16.3)$ & $8400(17.5)$ \\
\hline Injury or poisoning & $12977(42.1)$ & $12968(42.0)$ & $37601(42.2)$ & $17691(36.9)$ \\
\hline \multicolumn{5}{|l|}{ No of hospitalisations: } \\
\hline 0 & $24865(80.6)$ & $24962(80.9)$ & $70142(78.7)$ & $37656(78.6)$ \\
\hline 1 & $4095(13.3)$ & $4080(13.2)$ & $12329(13.8)$ & $6962(14.5)$ \\
\hline 2 & $1259(4.1)$ & $1193(3.9)$ & $4422(5.0)$ & $2125(4.4)$ \\
\hline$\geq 3$ & $624(2.0)$ & $608(2.0)$ & $2184(2.5)$ & $1146(2.4)$ \\
\hline \multicolumn{5}{|l|}{ Drugs: } \\
\hline Prescription acetaminophen & $9604(31.1)$ & $9504(30.8)$ & $23413(26.3)$ & $13577(28.4)$ \\
\hline Prescription NSAID & $7289(23.6)$ & $7187(23.3)$ & $19802(22.2)$ & $11610(24.2)$ \\
\hline ACE inhibitor & $18467(59.9)$ & $18377(59.6)$ & $55084(61.8)$ & $27632(57.7)$ \\
\hline Angiotensin II receptor blocker & $3523(11.4)$ & $3330(10.8)$ & $10850(12.2)$ & $4631(9.7)$ \\
\hline Thiazide diuretics & $5688(18.4)$ & $5486(17.8)$ & $15096(16.9)$ & $8134(17.0)$ \\
\hline Loop diuretics & $5690(18.4)$ & $5615(18.2)$ & $14593(16.4)$ & $8714(18.2)$ \\
\hline Potassium sparing diuretics & $2212(7.2)$ & $2153(7.0)$ & $5747(6.5)$ & $3453(7.2)$ \\
\hline Other diuretics & $1096(3.6)$ & $1060(3.4)$ & $2928(3.3)$ & $1775(3.7)$ \\
\hline$\beta$ blockers & $21658(70.2)$ & $21509(69.7)$ & $63559(71.4)$ & $33531(70.0)$ \\
\hline Calcium channel blockers & $8526(27.6)$ & $8295(26.9)$ & $22386(25.1)$ & $13701(28.6)$ \\
\hline Antibiotics & $13248(43.0)$ & $13034(42.3)$ & $36303(40.8)$ & 19835 (41.4) \\
\hline
\end{tabular}

$\mathrm{CABG}=$ coronary artery bypass graft, $\mathrm{PCTA}=$ percutaneous coronary intervention, NSAID=non-steroidal anti-inflammatory drug, $\mathrm{ACE}=$ angiotensin converting enzyme.

*Matching was to allow comparison of patients conditional on propensity score. 
Table 2| Comparison of new cases of diabetes and their matched controls, in the year before cohort entry. Values are numbers (percentages) of patients unless stated otherwise

\begin{tabular}{|c|c|c|}
\hline & \multicolumn{2}{|c|}{ New onset diabetes } \\
\hline & Cases $(n=3629)$ & Controls ( $n=35623$ ) \\
\hline \multicolumn{3}{|l|}{ Age (years): } \\
\hline $40-49$ & $294(8)$ & $2714(8)$ \\
\hline $50-59$ & $700(19)$ & $6928(19)$ \\
\hline $60-64$ & $423(12)$ & $4204(12)$ \\
\hline $65-69$ & $511(14)$ & $5222(15)$ \\
\hline $70-74$ & $581(16)$ & $5715(16)$ \\
\hline $75-79$ & $516(14)$ & $5072(14)$ \\
\hline$\geq 80$ & $604(17)$ & $5768(16)$ \\
\hline Women & $1324(36)$ & $12780(36)$ \\
\hline \multicolumn{3}{|c|}{ Events or procedures in year before cohort entry: } \\
\hline Myocardial infarction & $2421(67)$ & $23271(65)$ \\
\hline Stroke & $679(19)$ & $6661(19)$ \\
\hline CABG & $317(9)$ & $3580(10)$ \\
\hline PCTA & $618(17)$ & $6301(18)$ \\
\hline Hypertensive disease & $2169(60)$ & $19527(55)$ \\
\hline Hypercholesterolemia & $1034(28)$ & $11419(32)$ \\
\hline Peripheral vascular disease & $141(4)$ & $1094(3)$ \\
\hline Congestive heart failure & $780(21)$ & $5499(15)$ \\
\hline Injury or poisoning & $1395(38)$ & $13825(39)$ \\
\hline \multicolumn{3}{|l|}{ No of hospitalisations: } \\
\hline 0 & $2783(77)$ & $28084(79)$ \\
\hline 1 & $514(14)$ & $4852(14)$ \\
\hline 2 & $204(6)$ & $1755(5)$ \\
\hline$\geq 3$ & $128(4)$ & $932(3)$ \\
\hline \multicolumn{3}{|l|}{ Mean (IQR) No of drugs dispensed: } \\
\hline prescription acetaminophen & $1038(29)$ & $9594(27)$ \\
\hline Prescription NSAIDs & $914(25)$ & $8332(23)$ \\
\hline ACE inhibitors & $2283(63)$ & $21198(60)$ \\
\hline Angiotensin II receptor blockers & $455(13)$ & 3959 (11) \\
\hline Thiazide diuretics & $713(20)$ & $5865(16)$ \\
\hline Loop diuretics & $749(21)$ & $5386(15)$ \\
\hline Potassium sparing agents & $307(8)$ & $2342(7)$ \\
\hline Other diuretics & $133(4)$ & $1221(3)$ \\
\hline$\beta$ blockers & $2648(73)$ & $25378(71)$ \\
\hline Calcium channel blockers & $1120(31)$ & $8961(25)$ \\
\hline Antibiotics & $1583(44)$ & $14387(40)$ \\
\hline
\end{tabular}

$\mathrm{CABG}=$ coronary artery bypass graft, $\mathrm{PCTA}=$ percutaneous coronary intervention, IQR=interquartile range, NSAID=non-steroidal anti-inflammatory drug, $\mathrm{ACE}=$ angiotensin converting enzyme.

Among the 39252 patients in the study population, 35241 were prescribed statins at the time of their index date. 


\section{Figure}

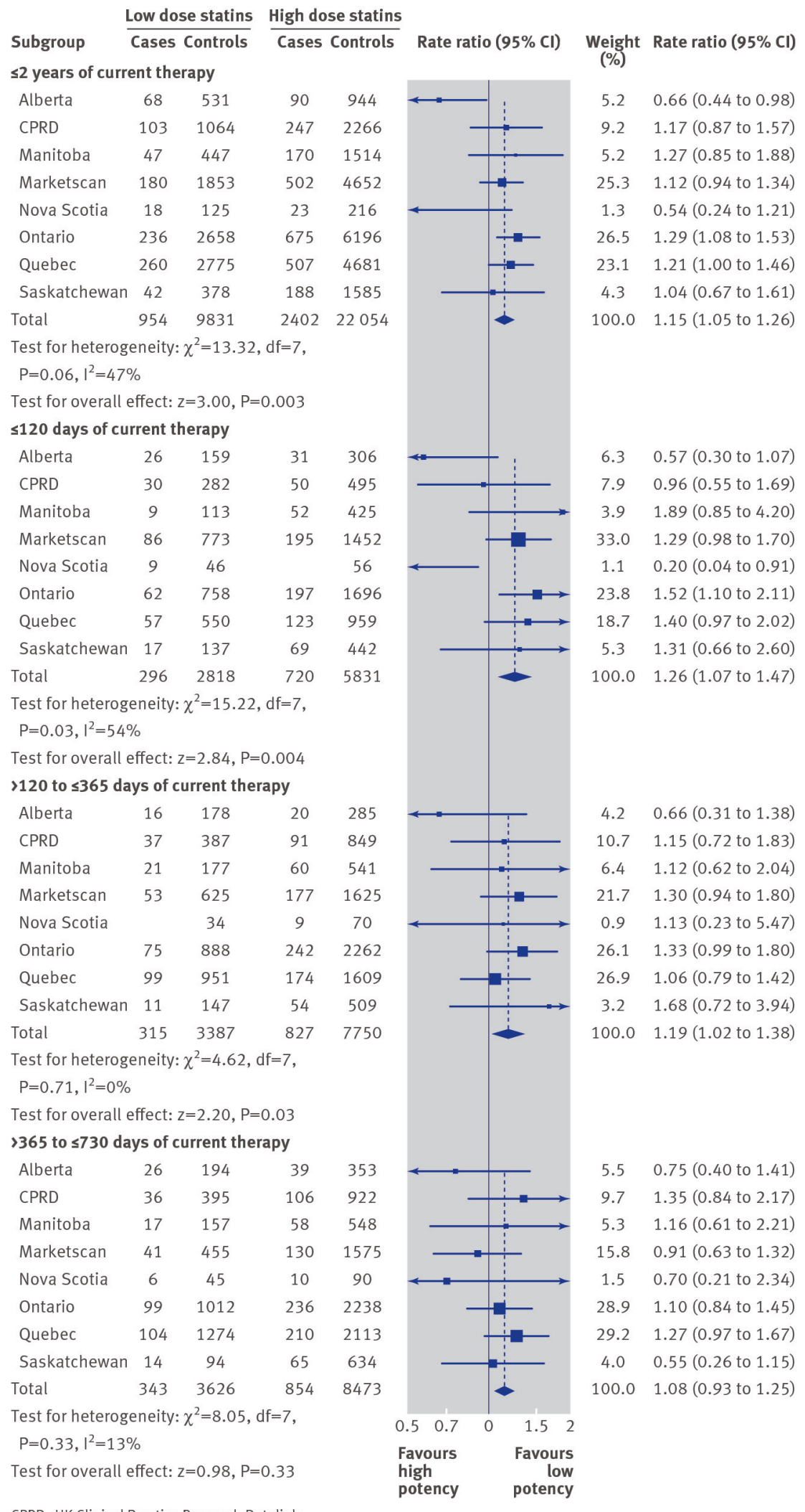

Rate ratios for new onset diabetes within two years of starting higher potency or lower potency statins after a major cardiovascular event or procedure (as-treated analysis) 